\title{
Complicaciones físicas del consumo de drogas recreativas
}

\author{
Eva Ojeda Rodríguez*, José Martínez Raga*, Miguel Castellano Gómez* *, \\ Bartolomé Pérez Gálvez**, Ana Sabater Ferragut*, Gaspar Cervera Martínez*** \\ *Unidad de Conductas Adictivas del Area 9 de Valencia. C. S. San Marcelino. Consellería de Sanidad, Generalitat Valenciana. \\ **Dirección General de Drogodependencias. Consellería de Bienestar Social, Generalitat Valenciana. \\ ** Unidad de Desintoxicación Hospitalaria. Servicio de Psiquiatría. Hospital Clínico Universitario, Valencia.
}

Enviar correspondencia: Dr. José Martínez Raga. Unidad de Conductas Adictivas, Área 9. Centro de Salud de San Marcelino. San Pio X, 32. 46017 Valencia (Spain) Tel. +34 96 3789496. Fax +34 96 3788254. E-mail: martinez_josrag@gva.es

\section{Resumen}

A lo largo de los últimos años se ha observado un aumento de las publicaciones en la literatura científica sobre las reacciones tóxicas, las complicaciones físicas y los fallecimientos asociados con el consumo de drogas recreativas, paralelo al aumento en la prevalencia de consumo de estas sustancias. Junto con las reacciones adversas que aparecen asociadas a la intoxicación aguda, el consumo de estas sustancias puede provocar complicaciones a nivel cardiovascular, accidentes cerebrovasculares, convulsiones, un cuadro de hiponatremia con edema cerebral o importantes alteraciones a nivel hepático. Sin embargo una de las complicaciones más graves que pueden desarrollarse es el de un síndrome hipertérmico que puede provocar coagulación intravascular diseminada, rabdomiolisis, e insuficiencia renal aguda. En el tratamiento de la intoxicación aguda o ante la ausencia de un antídoto específico el abordaje es fundamentalmente de tipo sintomático y de soporte.

Palabras claves: drogas recreativas; complicaciones médicas; síndrome hipertérmico; hepatotoxicidad; tratamiento.

\section{Summary}

In recent years there has been an increase in the publications in the scientific literature on the toxic reactions, physical complications and deaths associated with "recreations drug" use, parallel to increase in the prevalence of this drug consumption. In addition to the adverse reactions that can be observed associated with acute intoxication, use of these substances may cause cardiovascular complications, cerebrovascular accidents, convulsions, hyponatremia leading to cerebral aedema, as well as important hepatotoxic effects. However, one of the most severe complications that may develop is a hyperthermic syndrome that may evolve to diseminated intravascular coagulation, rhabdomyolysis, and acute renal failure. The treatment of acute intoxication, in light of the absence of a specific antidote the management is primarily symptomatic and supportive.

Key Words: recreational drugs; medical complications; hyperthermia; hepatotoxicity; treatment.

\section{INTRODUCCIÓN}

A lo largo de las dos últimas décadas se ha producido una expansión del abuso de las denominadas "drogas recreativas" particularmente en jóvenes.
Junto con este progresivo aumento del consumo, también han ido apareciendo un creciente número de informes sobre su toxicidad, así como descripciones de complicaciones graves relacionadas con estas sustancias. 
A la hora de valorar los efectos adversos del consumo de la MDMA hay que considerar que la mayoría de consumidores de éxtasis también usan simultáneamente otras sustancias. Hasta el momento existen muy escasos datos sistemáticos sobre la fenomenología de los estados inducidos por la MDMA, así como complicaciones sistémicas y neuropsiquiátricas. Así mismo, existen escasos datos epidemiológicos fiables sobre la comorbilidad psiquiátrica en consumidores de MDMA u otras drogas de diseño. La mayoría de datos provienen de estudios sobre casos únicos o sobre un número muy reducido de casos, o bien de estudios retrospectivos, con lo que por lo general se hace difícil descartar que en el desarrollo de las reacciones adversas participen mecanismos de tolerancia aguda o de sensibilización, la presencia de patología previa, el uso concomitante de otras drogas, o reacciones alérgicas o idiosincráticas. De hecho, en las revisiones bibliográficas se resalta la elevada psicopatología previa y el frecuente uso de otras substancias psicoactivas en los casos publicados de sujetos que habían desarrollado reacciones adversas asociadas al consumo de MDMA (Bango et al, 1998).

Las drogas de uso "recreativo" comprenden un grupo heterogéneo de sustancias. La variabilidad de los efectos es muy grande y obedece a diversos factores de susceptibilidad individual, farmacológicos e incluso culturales (Llopis et al, 2001). Uno de los primeros problemas que nos encontramos a la hora de valorar tanto los efectos agudos de este tipo de sustancias como su toxicidad y el tratamiento de los trastornos asociados con su consumo es el hecho de que nos encontramos, como ya se ha mencionado, ante un grupo muy amplio y diverso de sustancias, que aparecen reflejadas en la tabla 1.

Seguidamente vamos a revisar los efectos adversos de la sustancia a nivel físico, planteando finalmente las posibilidades de abordaje terapéutico conocidas hasta el momento. Por motivos de relevancia clínica y epidemiológica vamos a centrarnos fundamentalmente en la 3,4-metilenodioximetanfetamina (MDMA), conocida popularmente como éxtasis. Esta sustancia pertenece al grupo de las feniletilaminas, al igual que la 3,4-metilenodioxianfetamina (MDA, tenamfetamina, píldora del amor), la N-etil-3,4-metilenodioxianfetamina (MDE, MDEA, Eva), la metanfetamina (speed, ice), o la 4-metil-2,5-dimetoxianfetamina (DOM, STP) (Camí Morell, 1990).

\section{EL CONTEXTO DEL CONSUMO EN LA APARICIÓN DE COMPLICACIONES FÍSICAS}

Con toda seguridad la mayor incidencia de intoxicaciones y fallecimientos relacionados con el abuso de éxtasis en Europa obedece a las condiciones en que se consume tanto de tipo ambiental, grandes salas masificadas y con altas temperatura, como individuales, sensibilidad metabólica previa y nivel elevado durante horas de ejercicio- baile- con escasa reposición hídrica, todo ello sin menospreciar las características farmacológicas de la sustancia. Gran parte de las reacciones tóxicas descritas se han producido en un contexto de cambio cultural del consumo. El consumo masificado de MDMA en grandes fiestas pseudoclandestinas o macrodiscotecas de

Tabla 1: Drogas de síntesis más habituales en nuestro medio

- Ketamina

- Gammahidroxibutirato, GHB o éxtasis líquido

- Fenciclidina, PCP o polvo de ángel.

- Metanfetamina o speed.

- 3,4-metilenodioximetanfetamina (MDMA) o éxtasis. 
horario interminable, ha llevado a la aparición de una serie de casos notificados de reacciones adversas, intoxicaciones e incluso muertes. Por el contrario es curioso que durante la época de uso mistificado o terapéutico no se ha notificado un solo caso de reacciones adversas por éxtasis (Roig Traver, 1994; Schifano et al, 1998).

En los últimos 15 años se han consumido centenares de miles de dosis de MDMA en España sin observar una avalancha de consumidores con problemas de salud física o psíquica sobre los servicios sociosanitarios. Esto nos llevaría a una conclusión en extremo fácil y que evitaremos formular sin verificar la realidad. El elevado consumo de drogas de diseño no se corresponde con las aparentemente escasas repercusiones físicas, con lo que la MDMA se tiene como una sustancia poco peligrosa, falta de toxicidad y con escasos efectos residuales. Sin embargo, los efectos secundarios son conocidos y ampliamente descritos en la literatura internacional, remitiéndose por lo esencial a efectos simpaticomiméticos moderados.

Afirmaciones como estas, no exentas en muchas ocasiones de fanatismo, deben ser revisadas y puntualizadas para situar a la sustancia en su punto justo de equilibrio entre las drogas criminalizadas y las sustancias recreativas supuestamente inocuas.

La existencia de niveles elevados de otras drogas en sangre hace difícil atribuir una causalidad directa entre la muerte e ingestión de MDMA. Si bien algunos autores han Ilegado a señalar que el riesgo letal del éxtasis es extremadamente limitado, no se puede negar que este existe pero entre los cientos de miles de consumidores se han verificado muy pocos casos de muerte, muchos menos que en la mayor parte de actividades deportivas practicadas al aire libre (Seur, 1996). Recientemente se ha publicado un análisis de las muertes relacionadas con el consumo de éxtasis en Inglaterra y Gales entre los años 1997 y 2000 (Schifano et al, 2003), observándose que se produjeron un total de 81 muertes asociadas con esta sustancia, la mayoría de las cuales tuvieron lugar en el curso de fiestas o celebraciones y frecuentemente tras haber consumido varias sustancias simultáneamente.

\section{EFECTOS ADVERSOS O REACCIONES AGUDAS}

Bajo esta denominación se incluyen todas aquellas reacciones adversas que aparecen en el transcurso de los efectos agudos del consumo de MDMA y por lo tanto asociados a la intoxicación aguda, apareciendo entre los 15 minutos y las horas tras la ingesta. En varios estudios en los que se ha evaluado los efectos de la administración aguda de MDMA, observándose una variedad de reacciones adversas que aparecen resumidas en la tabla 2, alguna de las cuales persisten hasta 24 horas tras el consumo (Liester et al, 1992; Vollenweider et al, 1998). La mayor parte de estos problemas suelen desaparecer horas después del consumo y no suelen presentar mayores complicaciones.

\begin{tabular}{|l|}
\hline \begin{tabular}{c} 
Tabla 2: Reacciones de intoxicación \\
aguda asociada con el consumo de MDMA \\
- pérdida del apetito y de energía, con fatigabilidad \\
\hline - taquicardia, arritmias, junto con hipertensión arte- \\
rial
\end{tabular} \\
\hline - mayor sensibilidad al frío \\
- sofocos, aumento de la sudoración y sed \\
\hline - convulsiones \\
- hipertonía muscular, entumecimiento, y pareste- \\
sia, predominantemente en miembros inferiores, \\
\hline - pupilas dilatadas, visión borrosa y percepción dis- \\
torsionada de los colores
\end{tabular}

Los efectos adversos o reacciones agudas son menos frecuentes que los efectos colaterales y al igual que ellos aparecen con dosis 
moderadas en relación con determinada susceptibilidad individual, ya sea por patología preexistente o por idiosincrasia en personas sensibilizadas. De hecho, la aparición de reacciones agudas parece depender más de la susceptibilidad individual, de las circunstancias ambientales en que se ingiere y de la presencia de patologías previas, que de la dosis ingerida (Liester et al, 1992).

Dado que la MDMA acaba deplecionando las vesículas serotoninérgicas, el consumo continuado daría lugar a la aparición de un estado de sensibilización neurobiológica en el marco del cual aparecen estas reacciones y se desarrolla la tolerancia característica del éxtasis: tolerancia a los efectos positivos de éxtasis. Concretamente la pérdida de los efectos agradables de la sustancia que por otra parte son los buscados por el consumidor, debido a la falta de respuesta serotoninérgica ante nuevas administraciones de MDMA. Ante nuevos consumos no podrán aparecer nuevas descargas de serotonina en neuronas vacías de este neurotransmisor apareciendo únicamente los efectos adversos de tipo simpaticomimético y de descompensación del sistema serotoninérgico (McCann et al, 1998 y 2000).

Si bien tradicionalmente se ha pensado que la dopamina no parecía tener un papel relevante en la toxicidad aguda ni en la neurotoxicidad subaguda por MDMA, un estudio reciente ha mostrado claramente una importante y extensa neurotoxicidad dopaminérgica asociada con el uso ocasional de MDMA, que podría ser de gran importancia en la neurotoxicidad crónica (Ricaurte et al, 2002).

A nivel sistémico, se han descrito las siguientes reacciones agudas de toxicidad por MDMA (Burgess et al, 2000; Teter y Guthrie, 2001):

- Alteraciones cardiovasculares graves, como arritmias, asistolias, hipertensión y colapso cardiovascular.

- Accidentes cerebrovasculares: hemorragias intracraneales o infartos cerebrales que podrían ser atribuidos a una suscepti- bilidad individual al efecto hipertensivo de la MDMA.

- Convulsiones.

- Hipertermia aguda, coagulación intravascular diseminada, rabdomiolisis, e insuficiencia renal aguda.

- Han aparecido casos de anemia aplástica asociados al consumo de MDMA que mejoraban espontáneamente pasadas 7-9 semanas.

- Hepatotoxicidad, manifestada en forma de hepatitis, o insufuciencia hepática aguda, y caracterizada por ictericia, elevación aguda de la actividad de las transaminasas séricas y disminución de la actividad de la protrombina.

- Hiponatremia y secreción aumentada de hormona antidiuretica

\section{SÍNDROME HIPERTÉRMICO}

Entre las reacciones agudas el síndrome hipertérmico es la más frecuente y directamente relacionado con la letalidad por MDMA y tendría muchos aspectos comunes con la hipertermia maligna, el síndrome neuroléptico maligno y el golpe de calor. Sin embargo, en la literatura científica únicamente se han publicados 69 casos con reacción hipertérmica asociada al consumo de MDMA (Gowing et al, 2002). La mayor parte de los casos descritos de intoxicación por MDMA presentaban un cuadro clínico de sobredosis anfetamínica con hipertermia que evoluciona a fallo multiorgánico (Mechan et al, 2002). De hecho, desde hace muchos años es conocido un cuadro clínico de intoxicación por sobredosis de anfetaminas en el que se produce hipertermia grave seguida de coagulación intravascular diseminada, rabdomiolisis e insuficiencia renal, pero hasta el auge del consumo de éxtasis y la aparición de las primeras intoxicaciones era un cuadro muy escasamente descrito en la clínica.

A nivel experimental se ha demostrado que la MDMA es una sustancia hipertérmica 
para animales de laboratorio, pero también se ha visto que esta hipertermia corporal se modifica en relación a la temperatura ambiente de forma que ante temperaturas ambientales elevadas la hipertermia por MDMA es mayor y también su toxicidad, en cambio ante temperaturas ambientales bajas la respuesta hipertérmica central es menor de forma que la simple infusión de suero salino en ratas sometidas a elevada temperatura ambiente provoca una reacción de hipertermia central (Colado, 1998; McCann y Ricaurte, 1996).

En la práctica totalidad de los casos el cuadro de hipertermia aparece varias horas después de la ingestión de la droga en personas asistentes a fiestas o discotecas con gran concentración de personas y que habían empleado la sustancia con finalidad lúdica, sobre todo como droga de baile (Henry, 1992). En estas circunstancias la intensa actividad física bailando durante horas a causa de los efectos farmacológicos estimulantes junto con el ritmo sincopado de la música dance, la elevada temperatura de los locales nocturnos abarrotados y con escasa ventilación y las pérdidas hidroeléctricas mal compensadas por la escasa ingesta de líquidos, reducen la pérdida de calor corporal y potencian los efectos tóxicos directos de la MDMA sobre los mecanismos de termorregulación dando lugar a incremento de la temperatura corporal que puede llegar a los $42^{\circ} \mathrm{C}$. apareciendo el cuadro clínico de síndrome hipertérmico por MDMA (Llopis et al, 2001).

El cuadro tóxico agudo clásico aparece como una secuencia con inicio mediante un cuadro de agitación, taquicardia, hipertensión, midriasis y sudores. Este cuadro progresa rápidamente a una hipertermia superior a $40-41^{\circ} \mathrm{C}$, taquipnea, convulsiones y pérdida de conciencia (Gowing et al, 2002). La mayor parte de los cuadros de intoxicación se descubren ante esa pérdida de conciencia precedida o simultánea a un cuadro de convulsiones. En ese momento la posibilidad de intervención es mínima ya que han pasado varias horas desde el consumo con lo que el tratamiento sólo puede ser paliativo o sinto- mático, como más tarde veremos. En los casos más graves el cuadro evoluciona a una coagulación intravascular diseminada, rabdomiolisis, insuficiencia renal aguda y fracaso orgánico con resultado de muerte (Henry, 1992; Henry et al, 1992). El cuadro de intoxicación se acompaña de alteraciones hidroeléctricas con incremento de CPK, fosfatos, potasio y nitrógeno, así como descenso de bicarbonato en sangre, habitualmente asociado a leucocitosis e hiperglucemia (Colado y Lorenzo, 1995).

Se ha postulado que la elevación de la temperatura corporal y la intensa actividad física dan lugar a que el volumen del plasma se aproxime a la deshidratación con lo que la sangre comienza a coagularse allí donde más se la necesita apareciendo la coagulación intravascular diseminada por hipertermia, que no por alteración de factores plaquetarios (Shapiro, 1996). Otros autores describen la posibilidad de que las víctimas de hipertermia por éxtasis sufrieran al mismo tiempo una miopatía metabólica similar a la de las víctimas del golpe de calor por ejercicio físico (Henry, 1992; Henry et al, 1992). Sin embargo, lo que parece claro es que la aparición de hipertermia por MDMA no está directamente relacionada con la sustancia y que tendría que ver más con las circunstancias ambientales en que se consume y la predisposición individual.

\section{HEPATOTOXICIDAD}

Otra de las complicaciones agudas asociadas con el consumo de MDMA es el desarrollo de toxicidad hepática que puede dar lugar a ictericia, hepatomegalia, hipertransaminemias no justificadas e insuficiencia hepática aguda (Andreu et al, 1998). Hay al menos 39 casos publicados de pacientes que desarrollaron reacciones hepatotóxicas como consecuencia al consumo de MDMA (Gowing et al, 2002). La mayoría de los casos de hepatotoxicidad y hepatitis agudas por MDMA se resuelven espontáneamente sin que aparez- 
can indicadores de una evolución a cronicidad y sin poder hacer distinción entre los efectos de los derivados anfetamínicos y de los productos adulterantes de las pastillas (Jones y Simpson, 1999). Teniendo en cuenta la fuente ilegal de adquisición del éxtasis es posible que los adulterantes jueguen un importante papel en las génesis de estas complicaciones médicas (Steele et al, 1994). También se ha postulado que la reacción de hepatoxicidad podría producirse por una reacción de tipo inmunitario o una lesión secundaria a la hipertermia (Jones y Simpson, 1999).

El cuadro clínico suele empezar como una hepatitis aguda o subaguda con o sin colestasis, aunque la evolución va desde resolución rápida y espontánea, hasta casos de extrema gravedad que cursan con encefalopatía hepática, fallo hepático fulminante y muerte (Garbino et al, 2001; Lange-Brock et al, 2002). En los casos descritos en la serie estudiada por Henry y colaboradores en 1992 se observa el desarrollo de una hepatopatía varios días tras la ingestión de MDMA con ictericia colostática, necrosis hepatocelular e incremento de la AST en cifras superiores a 1500 unidades y ligera elevación de la GGT. En uno de los casos la consecuencia fue la muerte y en otro que había presentado tres episodios previos de hepatitis aguda grave tras tomar éxtasis precisó de transplante hepático.

La posibilidad de desarrollo de hepatopatía tóxica con necrosis también ha sido descrita como un complicación de la intoxicación por anfetaminas desconociéndose el mecanismo exacto de desarrollo (Beitia et al, 1999). Algunos estudios proponen la ingesta de éxtasis, como una de las etiologías a descartar en el contexto de una hepatitis aguda no viral en pacientes adultos jóvenes o adolescentes (Nuñez et al, 2002). En algunos casos se ha hecho necesario recurrir a un transplante hepático.

\section{ALTERACIONES CARDIOVASCULARES}

A nivel cardiovascular las propiedades simpaticomiméticas de la MDMA dan lugar a la aparición de taquicardia, vasoconstricción, hipertensión y anomalías del ritmo del tipo arritmias y extrasístoles además del ya descrito síndrome de coagulación intravascular diseminada. La frecuencia cardíaca muestra un incremento significativo entre la primera y segunda hora tras el consumo, para disminuir gradualmente hasta los niveles basales previos al consumo según los resultados del estudio de Grob en voluntarios sanos (Grob, 1998). En cuanto a las arritmias, cabe destacar la peligrosidad potencial del consumo de MDMA en personas que presenten un síndrome de preexcitación (por ejemplo un Wolff-Parkinson-White), ya que la taquicardia inducida por el fármaco puede favorecer la conducción aurículoventricular por la vía anómala en personas que hasta ese momento habían estado asintomáticas. Se cree que esta puede ser la causa de algunas muertes súbitas asociadas al consumo de MDMA (McCann et al, 1996).

En la serie de casos de intoxicación por MDMA estudiados por Henry y cols en 1992, algunos casos presentaron una prolongación del intervalo QT en el ECG como signo de riesgo de fibrilación ventricular, recomendando los autores que todo intoxicado por MDMA debe ser sistemáticamente monitorizado electrocardiográficamente por el grave riesgo de muerte súbita que entraña. De hecho, algunas de las muertes tras consumo de MDMA se han relacionado con la aparición de fibrilación ventricular y colapso cardiovascular (Henry, 1992; Henry et al, 1992; Steele et al, 1994).

\section{ACCIDENTE CEREBROVASCULAR}

El consumo de drogas recreativas como la MDMA se ha relacionado con distintos cuadros cerebrovasculares como la hemorragia subaracnoidea, la hemorragia intracraneal, el 
infarto cerebral y la trombosis del seno venoso cerebral (McEvoy et al, 2000; Rothwell y Grant, 1993). Como posibles mecanismos se han implicado, junto con la existencia de malformaciones vasculares subyacentes, la hipertensión aguda que produce la MDMA por sobrestimulación simpática, una posible inflamación de los vasos craneales, las alteraciones a nivel serotoninérgico, el incremento de la perfusión sanguínea a nivel cerebral y, también, la deshidratación que a menudo sufren los consumidores de éxtasis, que podría producir una trombosis cerebral.

\section{EDEMA CEREBRAL E HIPONATREMIA}

En 1994 se publicó el primer caso conocido de hiponatremia grave (Kessel, 1994). Desde entonces se han identificado múltiples casos de hiponatremina asociada a la MDMA, describiéndose que la MDMA puede causar edema cerebral o encefalopatía hiponatrémica, posiblemente secundarios a un síndrome de secreción inadecuada de hormona antidiurética (SIADH), tras consumo de MDMA junto a una excesiva ingesta de agua y que puede tener un resultado de muerte (Hartung et al, 2002). Aunque no se conoce el mecanismo exacto, se sabe que la MDMA puede estimular la secreción de la citada hormona (Fallon et al, 2002), pudiendo además provocar hiperprolactinemia y elevación en las concentraciones de ACTH. Los efectos simpaticomiméticos de esta sustancia asociados a la secreción inadecuada de hormona antidiurética unida a la deshidratación severa que aparece con la MDMA y que da lugar a la ingesta masiva de agua puede provocar una hiponatremia dilucional que a su vez puede llegar a causar un edema cerebral y graves lesiones encefálicas (Cherney et al, 2002).

\section{OTRAS COMPLICACIONES}

Debidos a los efectos estimulantes de la MDMA, así como de otras drogas recreativas $y$ al intenso ejercicio en el contexto del consumo se ha observado pérdida de apetito y de peso que puede mantenerse durante semanas (Liester et al, 1992). Por otro lado, estas sustancias se han asociado con trastornos del sueño, tanto de forma aguda como consecuencia a largo plazo, particularmente en consumidores crónicos (Parrott, 2001).

El consumo de MDMA se asocia también con la aparición de fatigabilidad y dolores musculares generales, con tensión muscular a nivel mandibular que a su vez trae como resultado bruxismo y trismus (Vollenweider et al, 1998). Así mismo, se han descrito lesiones a nivel bucal del tipo serostomía química y erosiones dentales como consecuencia de estos efectos mandibulares (Duxbury, 1993).

Se ha sugerido que el consumo de MDMA podría aumentar el riesgo de desarrollar enfermedad de Parkinson debido a un efectos neurotóxico sobre células serotoninérgicas y dopaminérgicas (Ricaurte et al, 2002). De hecho, se han observado algunos casos de parkinsonismo tras la ingesta de éxtasis (Mintzer et al, 1999).

\section{TRATAMIENTO DE LA INTOXICACIÓN AGUDA}

La ausencia de un antídoto específico hace que el tratamiento sea fundamentalmente sintomático y de soporte, con el máximo de efectividad dentro de las dos primeras horas post-consumo. El cuadro sintomático, tal como se ha mencionado anteriormente, es muy similar al de un "golpe de calor" y guarda grandes semejanzas con el síndrome serotoninérgico. Tendiendo en cuenta la gravedad del cuadro, es conveniente que la intoxicación aguda sea tratada en un centro hospitalario con Unidad de Vigilancia Intensiva. El 
tratamiento se basa en medidas de soporte pero con ciertas peculiaridades:

- Lavado gástrico siempre que se esté actuando en las primeras dos horas, ya que pasado este tiempo pierde su eficacia.

- Control de constantes vitales, con especial atención en la frecuencia cardíaca, respiración y tensión arterial.

- Rehidratación con sueros hidrosalinos. Es necesario aclarar las recomendaciones difundidas habitualmente de beber abundante agua ante los síntomas del golpe de calor. Para evitar el riesgo de edema cerebral es más conveniente tomar bebidas isotónicas o sueros salinos que reducen el riesgo de absorción celular del agua sobrante y garantizan la reposición del equilibrio hidroeléctrico (Rieder, 2000; Shapiro, 1996).

- Provocar el aumento de la eliminación del tóxico en orina mediante la acidificación de la misma. Sin embargo, hay que tener en cuenta que al acidificar la orina hay riesgo de mioglobinuria y consiguiente fracaso renal.

- Controlar la hipertermia fundamentalmente mediante medidas de soporte. A menudo se recomienda la utilización del dantrolene para el tratamiento de la hipertermia secundaria al consumo de MDMA, si bien su utilización continúa siendo controvertida (Larner, 1993; Teter y Guthrie, 2001).

- Control sintomático de la tensión arterial mediante medicación apropiada.

- Tratamiento de las convulsiones.

- Evitar los estímulos externos y administración de haloperidol o diazepan en el caso de que fuera necesaria para la agitación.

\section{CONCLUSIONES}

El consumo de "drogas recreativas" se ha asociado con diversas complicaciones físicas, algunas de las cuales de gran gravedad. Sin embargo, el número global de casos publica- dos en la literatura científica es relativamente escaso, particularmente si tenemos en cuenta la elevada prevalencia de éxtasis y de otras drogas de síntesis. Esto podría reflejar una baja incidencia de efectos adversos graves asociados con el consumo de este grupo de sustancias o bien que estos no son debidamente identificados. Sin embargo, la importancia de las consecuencias sobre la salud de las "drogas recreativas" viene dada no sólo porque suelen aparecer en jóvenes, sino también por lo impredecibles que son y por ser potencialmente letales. En cualquier caso, el personal sanitario, especialmente en los Servicios de Urgencias necesitan conocer los efectos adversos debidos al consumo de drogas de síntesis para una mejor identificación de los casos y con ello poder aplicar el abordaje terapéutico más apropiado. Son necesarios estudios epidemiológicos adecuadamente diseñados para establecer la incidencia real de las complicaciones físicas del consumo de "drogas recreativas".

\section{REFERENCIAS}

1. Andreu V, Mas A, Bruguera M, et al. Ecstasy: a common cause of severe acute hepatotoxicity. J Hepatol 1998; 29:394-397.

2. Bango J, Fadón P, Mata F, Rubio G, SantoDomingo J. Trastornos psiquiátricos y consumo de MDMA (éxtasis): revisión de casos. Actas Luso-Esp Neurol Psiquiatr 1998; 26:260-263.

3. Beitia G, Cobreros A, Sainz L, Cenarruzabeitia E. 3,4-Methylenedioxymethamphetamine (ecstasy)-induced hepatotoxicity: effect on cytosolic calcium signals in isolated hepatocytes. Liver 1999; 19:234-241.

4. Burgess C, O'Donohoe A, Gill M. Agony and ecstasy: a review of MDMA effects and toxicity. Eur Psychiatry 2000; 15:287-294.

5. Camí Morell J. Síntesis clandestina de drogas: pasado, presente y futuro. Med Clin (Barc) 1990; 95:344-347.

6. Colado MI, Lorenzo P. MDMA: farmacología y toxicología. En: Bobes J.: Éxtasis, aspectos farmacológicos, psiquiátricos y médico legales. 
Pp 1-46. Monografías en neurociencias n15. Ed Neurociencias. Barcelona 1995.

7. Colado MI. Neurotoxicidad inducida por MDMA y su prevención farmacológica. En: Bobes y cols. Éxtasis (MDMA). Un abordaje comprehensivo. Tomo I. Pp 41-73. Colección Psiquiatría médica. Ed Masson S.A. Barcelona 1998.

8. Cherney DZ, Davids MR, Halperin ML. Acute hyponatremia and ecstasy: insights from a quantitative analysis. OJM 2002; 95:475-483.

9. Duxbury A.J. Ecstasy dental implications. Br Dent J 1993; 175:38.

10. Fallon JK, Shah D, Kicman AT, et al. Action of MDMA (ecstasy) and its metabolites on arginine vasopressin release. Ann N Y Acad Sci 2002; 965:399-409.

11. Garbino J, Henry JA, Mentha G, Romand JA. Ecstasy ingestión and fulminant hepatic failure: liver transplantation to be considered as a last therapeutic option. Vet Hum Toxicol 2001; 43:99-102.

12. Gowing LR, Henry-Edwards SM, Irvine RJ, Ali RL.The health effects of ecstasy: a literature review. Drug Alcohol Rev 2002; 21:53-63.

13. Grob CS. Investigación humana con MDMA. En Bobes y cols. Éxtasis: Un abordaje comprehensivo. Tomo I. Pp 99-106. Colección Psiquiatría médica. Ed Masson S.A. Barcelona 1998.

14. Hartung TK, Schofield E, Short Al, Parr MJ, Henry JA. Hyponatraemic states following 3,4methylenedioxymethamphetamine (MDMA, 'ecstasy') ingestion. QJM 2002 ; 95: 431-437.

15. Henry JA. Ecstasy and the dance of death. Severe reactions are unpredictible. BMJ 1992; 305:5-6.

16. Henry JA, Jeffreys KJ, Dawling S. Toxicity and deaths from 3,4-methylenedioxymethamphetamine. Lancet 1992; 340:384-387.

17. Jones AL, Simpson KJ. Review article: mechanisms and management of hepatotoxicity in Ecstasy (MDMA) and amphetamine intoxications. Aliment Pharmacol Ther 1999; 13:129133.

18. Kessel B. Hyponatraemia after ingestion of ecstasy. BMJ 1994; 308:414.

19. Lange-Brock N, Berg T, Muller AR, et al. Acute liver failure following the use of ecstasy. Z Gastroenterol 2002; 40:581-586.

20. Larner AJ. Dantrolene and ecstasy. Anesthesia 1993;48;179-180.
21. Levine AJ, Drew S, Rees GM. Ecstasy induce penumomediastinum. J R Soc Med 1993; 86:232-233.

22. Liester MB, Grob CS, Brabo GL, Walsh RN. Phenomenology and sequelae of 3,4-methylnedioxymethamphetamine use. J Nerv Ment Dis 1992; 180:345-352.

23. Llopis JJ, Farré M, Martínez-Raga J. Las drogas de diseño: clínica y tratamiento del abuso de éxtasis. En: Giner F, Cervera G (eds.) Trastornos Adictivos. Drogodependencias: clínica y tratamientos psicobiológicos. Generalitat Valenciana. Valencia, 2001; pp.405-438.

24. McCann U, Slate SO, Ricaurte GA. Adverse reactions with MDMA. Drug Saf 1996; 15:107115.

25. McCann UD, Ricaurte G. Aproximación a la neurobiología y neurotoxicidad comparada inducida por la MDMA. En Bobes y cols. Éxtasis (MDMA). Un abordaje comprehensivo. Tomo I.Pp 89-98. Colección Psiquiatría médica. Ed Masson S.A. Barcelona 1998.

26. McCann UD, Szabo Z, Scheffel U, Dannals RF, Ricaurte GA. Positron emission tomographic evidence of toxic effect of MDMA (AEcstasy@) on brain serotonin neurons in human beings. Lancet 1998; 352:1433-1437.

27. McCann UD, Eligulashvili V, Ricaurte GA. (+/) 3,4-Methylenedioxymethamphetamine ('Ecstasy')-induced serotonin neurotoxicity: clinical studies. Neuropsychobiology 2000; 42:11-6.

28. McEvoy AW, Kitchen ND, Thomas DG. Intracerebral haemorrhage and drug abuse in young adults. Br J Neurosurg 2000; 14:449-454.

29. Mechan AO, Esteban B, O'Shea E, Elliott JM, Colado MI, Green AR. The pharmacology of the acute hyperthermic response that follows administration of 3,4-methylenedioxymethamphetamine (MDMA, 'ecstasy') to rats. $\mathrm{Br} \mathrm{J}$ Pharmacol 2002;135:170-180.

30. Mintzer S, Hickenbottom S, Gilman S. Parkinsonism after taking ecstasy. N Engl J Med 1999; 340:1443.

31. Núñez O, Banares R, Barrio J, et al. Variabilidad de la expresión clínica de la toxicidad hepática por éxtasis. Gastroenterol Hepatol 2002; 25:497-500.

32. Parrott AC. Human psychopharmacology of Ecstasy (MDMA): a review of 15 years of empirical research. Hum Psychopharmacol 2001; 16:557-577. 
33. Ricaurte GA, Yuan J, Hatzidimitriou G, Cord BJ, McCann UD. Severe dopaminergic neurotoxicity in primates after a common recreational dose regimen of MDMA ("ecstasy"). Science 2002; 297:2260-2263.

34. Rieder MJ. Some light from the heat: implications of rave parties for clinicians. CMAJ 2000; 162:1829-30.

35. Roig Traver, A: El Éxtasis, una revisión del MDMA y demás feniletilaminas psicoactivas. Libro de actas de Socidrogalcohol XVII Jornadas Nacionales. Diputación de Valencia. Área de Salud Pública.1994; pp 541-561.

36. Rothwell, PM, Grant R. Central nervous sinus thrombosis induced by ecstasy. J Neurol Neurosug Psychiatry 1993; 56:1035.

37. Schifano F, Di Furia L, Forza G, Minicuci N, Bricolo R. MDMA ('ecstasy') consumption in the context of polydrug abuse: a report on 150 patients. Drug Alcohol Depend 1998; 52:85-90.

38. Schifano F, Oyefeso A, Webb L, Pollard M, Corkery J, Ghodse AH. Review of deaths related to taking ecstasy, England and Wales, 1997_2000. BMJ 2003; 326:80-81.

39. Seur C. Riduzione del danno nei rave party e tratamento delle complicación psicopatologiche. International Conferenze Ecstasy e Sostenza Psichedeliche. Ed Verso I'Utopia. Bolonia 1996; pp 116-146.

40. Shapiro H. A 16 Ecstasy update. Information for drug workers from ISDD. Drug Link photocopiable factsheet. ISDD, 1996.

41. Steele TD, McCann UD, Ricaurte GA. 3,4Methylenedioxymmethamphetamine: pharmacology and toxicology in animals and humans. Addiction 1994; 89:539-551.

42. Teter CJ, Guthrie SK. A comprehensive review of MDMA and GHB: two common club drugs. Pharmacotherapy $2001 ; 21: 1486-1513$.

43. Vollenweider FX, Gamma A, Liechti M, Huber T. Psychological and cardiovascular effects and short-term sequelae of MDMA (Aecstasy@) in MDMA-naïve healthy volunteers. Neuropsychopharmacology 1998; 19:241-251. 